\title{
A Machine Learning Approach to Discriminate Between Soft and Hard Bone Tissues Using Drilling Sounds
}

\author{
Vahid Zakeri*, Francesco Fabri, Masashi Karasawa, and Antony J Hodgson \\ University of British Columbia, Vancouver, Canada \\ *vahid.zakeri@mech.ubc.ca
}

\begin{abstract}
Bone drilling is conducted in many surgical disciplines such as orthopedics, maxillofacial, and spine surgery. Most of these procedures involve drilling of different bone materials including hard (cortical) and soft (cancellous) tissues. Identifying these tissues is essential for surgeons to minimize damage to underlying nerves and vessels.

The sound signal generated during drilling is a valuable source of information that could potentially be employed. Such sounds can be captured readily and easily through non-contact sensors. Therefore, our goal in this preliminary study is to investigate whether drilling sounds can enable us to distinguish between cortical and cancellous tissues.

A bovine tibial bone was drilled, and the cortical and cancellous drilling sounds were captured. Each sound record was divided into small windows with a length of 50 ms and a 50\% overlap. The window length was selected small, because our intended longer-term application is to provide the surgeon with near-real-time feedback. Short time Fourier Transform (STFT) coefficients were extracted from each window and were averaged accordingly to obtain $\mathrm{p}$ features. A support vector machine (SVM) algorithm was used for classification, and its accuracy was evaluated for different number of features $(p)$. Two training/testing scenarios were considered, atlas (ATL) and leave-one-out (LOO).

The total accuracies for ATL and LOO were $100 \%$ and $93.8 \%$ respectively obtained for $p=128$. Our study on a single specimen demonstrated that it is possible to discriminate between cortical and cancellous bones based on relatively short $50 \mathrm{~ms}$ windows of drilling sounds.
\end{abstract}




\section{Introduction}

Bone drilling is performed in many surgical procedures including orthopedics, maxillofacial, and spine surgeries to prepare holes for screws and other anchoring or fixation devices. Most of these procedures involve drilling through relatively harder (cortical) and softer (cancellous) tissues. To minimize damage to underlying nerves and vessels, drilling should be stopped after passing through the first cortex and the cancellous zone and before breaching the second cortex (Allotta 1997) (Hu 2014). During such procedures, surgeons rely on tactile and visual cues to identify the tissue type through which they are cutting. However, because of property and process variations, these cues may not be adequate (Praamsma 2008).

There have been reports in the literature on various devices for identifying different bone tissues such as a hand-held drilling tool for orthopedic surgeries (Allotta 1997) and a robotic system for pedicle screw insertion (Hu 2014). Most of these approaches have employed some form of force sensing that requires modifying the surgical drill, potentially making the system more cumbersome or markedly more expensive. Therefore, it is useful to look for other sources of information that can provide feedback about the drilling procedure. The sound signal generated during drilling is a valuable source of information that could potentially be employed. Such sounds can be captured readily and easily through non-contact sensors. Therefore, our goal in this preliminary study is to investigate whether drilling sounds can enable us to distinguish between cortical and cancellous tissues.

\section{Materials and Methods}

The experiment was performed on a bovine tibial bone that was fixed in a vise (Figure 1). A Conmed Linvatec Hall Mpower2, Pro6202M surgical handpiece was used to drill the bone. The drilling was repeated three times for each tissue, and the sound was recorded with the sampling frequency of $20 \mathrm{kHz}$. The duration of recording was $\sim 9-13 \mathrm{~s}$ in the cortical bone and $\sim 3-6 \mathrm{~s}$ in the cancellous bone. Each recording was windowed with a 1000-point Hamming window and a 50\% overlap. The window length was selected to represent a relatively short period of time $(50 \mathrm{~ms})$ because our intended longer-term application is to provide the surgeon with near-real-time feedback. The data of each window were transformed to the frequency domain using the fast Fourier transform (FFT). The first 512 FFT coefficients were bin-averaged to obtain $p$ number of features to be used for classification. Each feature then was scaled linearly to the interval $[-1,1]$. The number of features was varied as $p=2^{n}(n=1,2, \ldots, 9)$, and for each $p$, the total classification accuracy was obtained.

To classify the sound recordings, we used a support vector machine (SVM) approach. SVM is a powerful machine learning algorithm that classifies data into two classes by finding a hyper-plane that maximizes the separation between them. It can be formulated as a convex max-min optimization problem (Cortes 1995). In this study, we used SVM with a radial basis function (RBF) kernel, because of its capability in handling nonlinear data and fewer numerical difficulties (Hsu 2003).

To use SVM for classification, we separated the data into two sets with no overlap, training set and testing set. We evaluated two training/testing scenarios: atlas (ATL) and leave-one-out (LOO). In ATL, the data of all recordings from both bone types were considered together. Half of these data were randomly and uniformly selected for training, and the other half were used for testing. This random training/testing procedure was repeated 100 times to avoid bias and overfitting. In the LOO scenario, we chose the training data from two of the recordings, and the testing data from the remaining (third) recording. For both ATL and LOO, SVM models were developed using different number of features $(p)$. The kernel parameters were adjusted using a 5-fold cross validation. The analysis was performed using the MATLAB platform. 


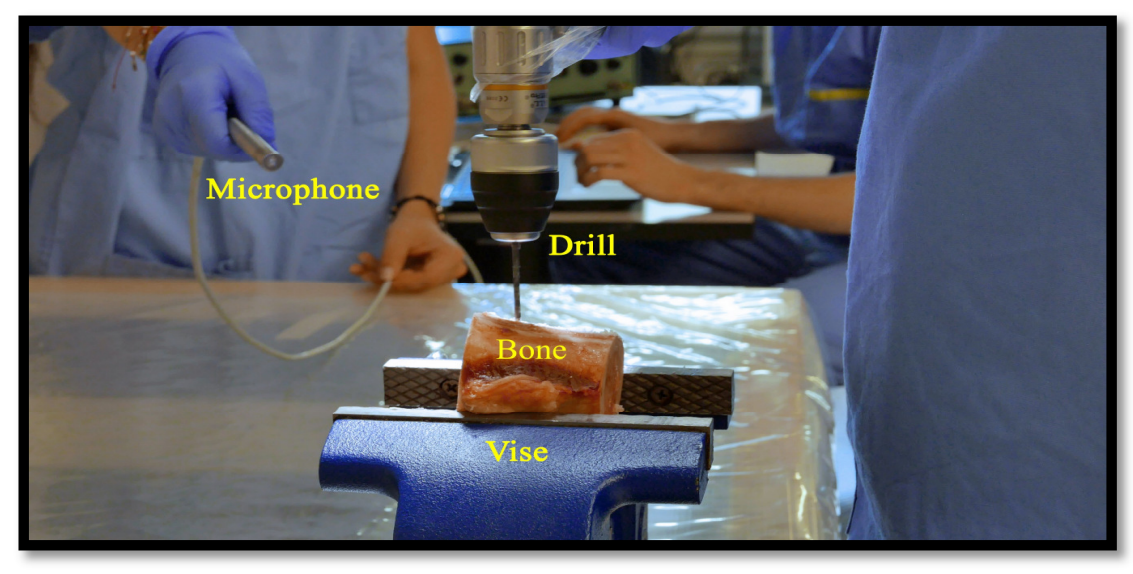

Figure 1: The experimental setup

\section{Results}

Figure 2 shows the total accuracies of ATL and LOO scenarios for different number of features (p). As we can see, the accuracies were first increased and then remained constant by incrementing $\mathrm{p}$. At $\mathrm{p}=128$, the accuracies of both scenarios have reached their steady states; i.e. $100 \%$ for ATL, and 93.8\% for LOO. For this number of features, Table I indicates the cortical and cancellous accuracies. In the ATL scenario, the classification was perfect, while in the LOO scenario, cortical bone was still correctly identified $100 \%$ of the time, but nearly a fifth of the cancellous samples were misidentified as cortical.

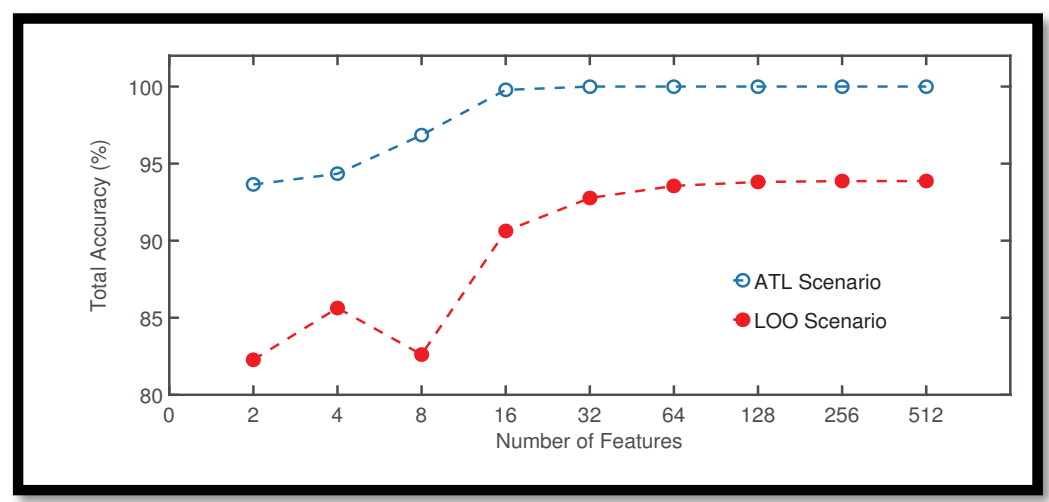

Figure 2: The effect of different number of features on the total accuracy

\begin{tabular}{llc}
\hline \hline & ATL & LOO \\
\hline Cortical Accuracy & $100 \%$ & $100 \%$ \\
Cancellous Accuracy & $100 \%$ & $83.2 \%$ \\
Total Accuracy & $100 \%$ & $93.8 \%$ \\
\hline \hline
\end{tabular}

Table 1: The Classification Results for ATL and LOO Scenarios $(p=128)$ 


\section{Discussion}

This experiment on a single specimen demonstrated that it is possible to discriminate between cortical and cancellous bones based on relatively short $50 \mathrm{~ms}$ windows of drilling sounds. The cortical bone was reliably identified, and the cancellous bone had a relatively low identification error. The primary limitation is that we have only evaluated performance of the classifier when trained on the specific sample being drilled, and so have not yet evaluated the generalizability of the performance of this classification approach to different specimens, which would be a more realistic use scenario.

Sound signals have been used for material classification in similar applications in other fields such as dentistry (Zakeri 2014), rock drilling (Vardhan 2009), and tool-wear monitoring (Trabelsi 1991). The closest study to ours was conducted by (Dai 2014), in which drilling sounds and wavelet-based features were used to identify the type of bone being drilled. They mentioned that the approach was capable of recognizing cortical and cancellous bone, but did not report classification accuracies.

In future, we plan to investigate the generalizability of this technique across different bone samples and users, and, if sufficiently promising, to evaluate the value of providing a real-time tissue property classification on performance of selected surgical procedures that involve bone drillings.

\section{Acknowledgement}

This work was supported by funds provided through the Michael Smith Foundation for Health Research, British Columbia, Canada.

\section{References}

Allotta B, Giacalone G, Rinaldi L (1997) A hand-held drilling tool for orthopedic surgery. IEEE/ASME Trans Mechatronics 2:218-229.

Hu Y, Jin H, Zhang L, et al (2014) State recognition of pedicle drilling with force sensing in a robotic spinal surgical system. IEEE/ASME Trans Mechatronics 19:357-365.

Praamsma M, Carnahan H, Backstein D, et al (2008) Drilling sounds are used by surgeons and intermediate residents, but not novice orthopedic trainees, to guide drilling motions. Can J Surg 51:442-446.

Cortes C, Vapnik V (1995) Support-vector networks. Mach Learn 20:273-297.

Hsu C, Chang C, Lin C (2003) A practical guide to support vector classification.

Zakeri V, Arzanpour S, Chehroudi B (2014) Discrimination of tooth layers and dental restorative materials using cutting sounds. IEEE J Biomed Heal Informatics 19:571-580.

Vardhan H, Adhikari GR, Govinda Raj M (2009) Estimating rock properties using sound levels produced during drilling. Int J Rock Mech Min Sci 46:604-612.

Trabelsi H, Jr EK-A (1991) Pattern-recognition analysis of sound radiation in metal cutting. Int J Adv Manuf Technol 6:220-231.

Dai Y, Xue Y, Zhang J (2014) Condition monitoring based on sound feature extraction during bone drilling process. In: Chinese Control Conf. pp 7317-7322 